\title{
Method of Expanding Measurement Limits of Digital Dynamometer in Testing Strength of Moulded Peat Products
}

\author{
Alexander Yablonev $^{1, *}$, and Anna Guseva ${ }^{1}$ \\ ${ }^{1}$ Tver State Technical University (TvSTU), 170026, Afanasy Nikitin emb., 22, Tver, Russia
}

\begin{abstract}
The work is carried out to survey operating quality of sod peat produced by milling-forming machines in the field. The surveillance will help make necessary changes in mining machine designs and operating modes in a timely manner. One of the most important quality parameters of sod peat is its bending strength. To evaluate it, Tver State Technical University has developed a destruction test bench. The test bench is equipped with a digital dynamometer and a strain-gauge bracket torecord the destructive load. However, the instruments are designed for $1 \mathrm{kN}$ maximum load. The bending tests of moulded peat products conducted by the authors showed that the destructive load often exceeds $1 \mathrm{kN}$, which meant that the generation and measurement limits of destructive load have to be expanded within the range of equipment being available. The problem has been solved by installing additional elements in the straingauge bracket and making a calibration chart. The method can expand the generation and measurement limits of destructive load by more than $50 \%$.
\end{abstract}

\section{Introduction}

Production of high-quality sod peat directly depends on peat raw characteristics, mining machine parameters, dispersion and moulding modes [1, 2]. The proper production parameters can significantly reduce energy consumption and peat loss during production [3, 4]. The major method to survey product quality is to check the mechanical properties of sod peat at the conditioned moisture stage ( $w \approx 33 \%$ ), i.e. to use the so-called "follow-ups". Although these follow-ups are really remote in time from the direct extraction of peat for up to 30 days (the drying time of the extracted sod in the field), nevertheless, this is the most reliable parameter, which can be quickly applied to change the machine and entire technological process parameters. The sod density, usually determined with the volumeweight method, serves as an indicator of a fuel calorific value. The ultimate bending strength of a sod shows the peat preservation during all subsequent technological operations after direct moulding and laying on the field for drying [5,6]. Solid fuel is commonly tested for bending strength by methods of sod destruction with concentrated load on special hydraulic test benches. In this case, the destruction load is evaluated by the hydraulic pressure in the test bench system [7,8]. Since the hydraulic test bench is not mobile and the

\footnotetext{
*Corresponding author: alvovich@mail.ru
} 
pressure results obtained in the main line depend on the system inertia, a new test bench to test destruction of moulded products, including peat, has been developed at Tver State Technical University. The test bench has a mechanical drive and digital strain gauges, designed to generate and measure $1 \mathrm{kN}$ destructive load. The tool having small dimensions can give very accurate results. In addition, the authors have worked out its application in testing sod peat. However, in the course of the work, the authors faced the challenge, that the destruction of some sod peat samples required a load exceeding $1 \mathrm{kN}$. This load could be provided by the metal test bench structure (the test bench is designed to create a destruction load of up to $5 \mathrm{kN}$ ), but could not be with stood by the strain gauges, measuring sensor in the form of a strain-gauge $S$-shaped bracket and a system unit of a digital dynamometer. Therefore, the task was to expand the limits of measuring the destruction load with the available equipment.

\section{Method used}

Digital dynamometer DOU-3-1I, installed on the test bench, measures the concentrated load with a strain gauge, $S$-shaped bracket, in compression and tension tests. Inside the bracket there are strain resistors, glued to the bracket body and joined by a bridge connection. Tension occurs in the connection diagonals at the slightest deformation of the bracket body. The tension value is detected by the system unit converting an analog signal to a digital one. The latter is displayed as a load on the system unit. Thus, the device operation is based on Hooke's law and the system is described by a certain elastic coefficient, which is a coefficient of load and deformation proportionality. This leads to the conclusion that it is necessary to increase the elastic coefficient of the strain gauge bracket in order to measure a greater load. In this case, the system unit display will not reflect the true but relative load (under estimated due to the system elasticity increase). The ratio between the true and relative loads in such cases is determined by the calibration chart.

The task of increasing strain-gauge bracket elasticity is fulfilled by fitting and mounting additional metal spacers, made of M14 nuts into the bracket body. Adjusting the height of the nuts ensures their installing without a gap between the bracket body and shanks (fig. 1).

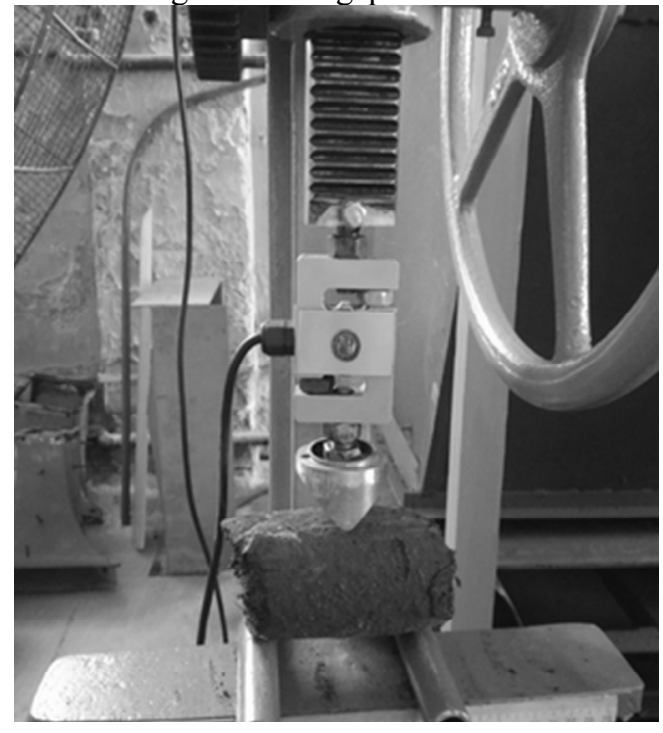

Fig. 1. Installing additional elements in the strain-gauge bracket body. 
The nut ends are ground and polished to improve the system accuracy. As a result, heavy loads generated by the test bench lead to the smaller bracket deformation and, consequently, to the smaller relative load displayed on the system unit. Such structure may be referred to as "clamped" strain-gauge bracket.

A calibration chart is made with a method developed for loading the test bench handwheel with the same calibrated loads in free and "clamped" states of the strain gauge bracket. In this case, the dynamometer tip rests against a hard metal disk being almost rigid under such loads (fig. 2).

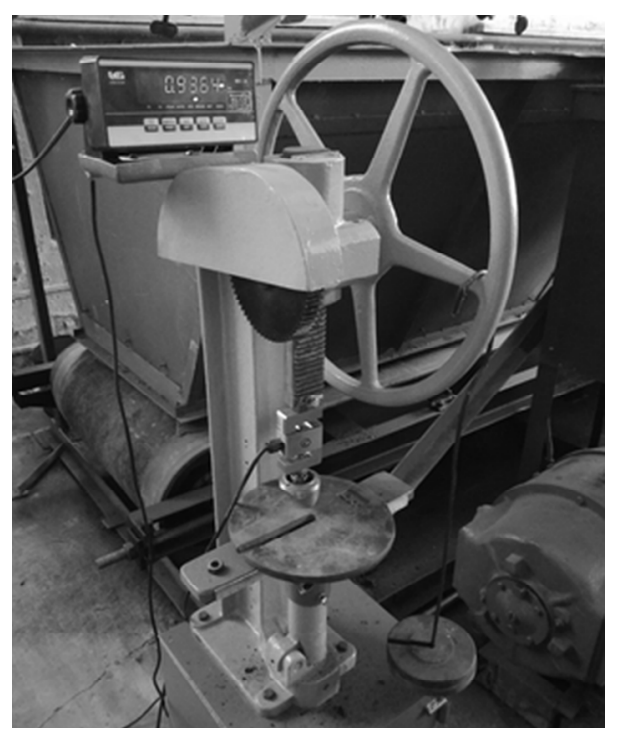

Fig. 2. The process of strain-gauge bracket calibration with calibrated weights.

The digital dynamometer displays the load generated by the test bench with the same calibrated loads when the strain-gauge bracket is free and clamped.

Fig. 2 shows, that the arm of force, creating the moment on the test bench, is chosen as maximum (loads are suspended on the handwheel periphery) to increase the accuracy in the subsequent reproduction of experimental results.

\section{Results and discussion}

Based on the research done we made a calibration chart of the dependence of true load $F$ on relative load $F_{\mathrm{o}}$ (under estimated) due to the strain-gauge bracket "clamping", fair for digital dynamometer DOU-3-1I (fig. 3). These chart data were reproduced, when the tests were repeated three times. 


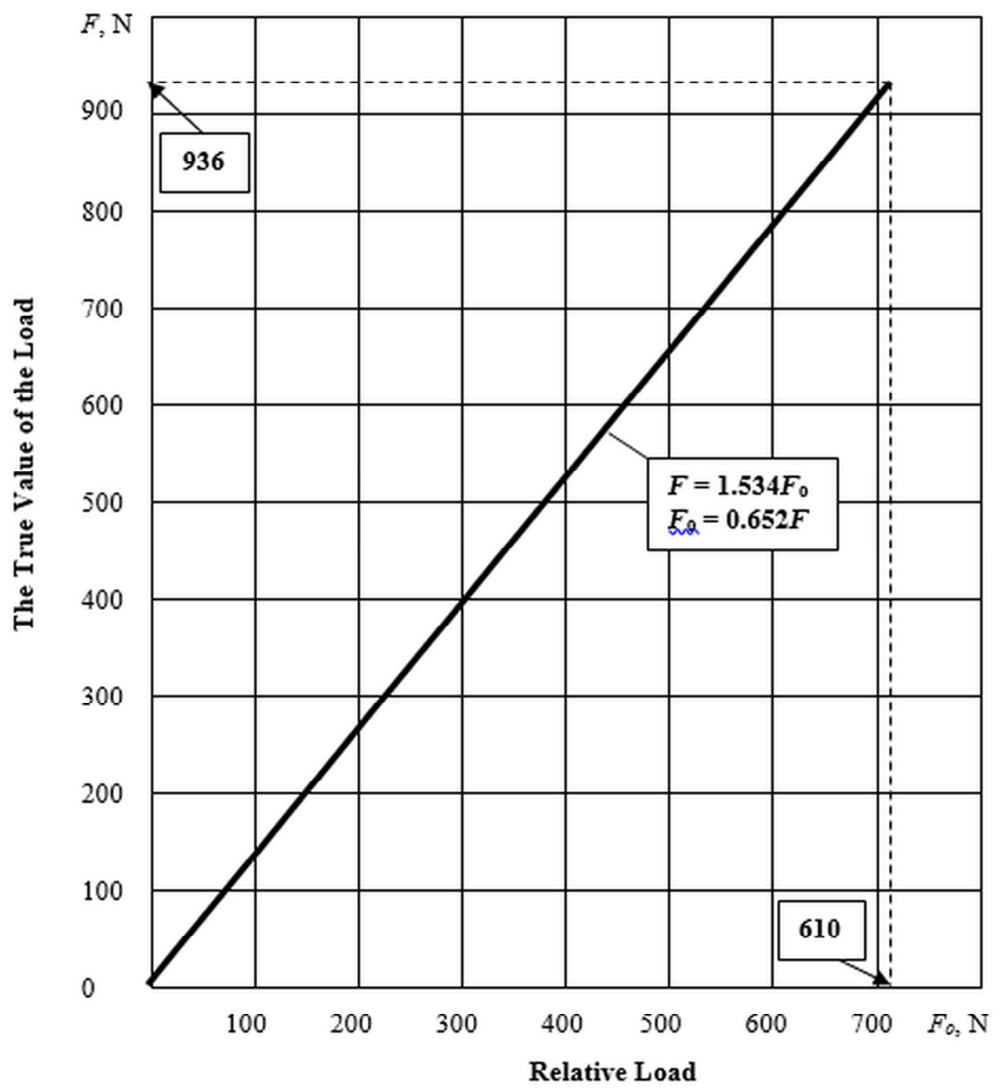

Fig. 3. A calibration chart.

Using the resulting chart and calibration equations placed on the graph field, we could easily convert the values of the relative load, when the strain-gauge bracket was clamped, to the true value, when it was free.

The analysis of the calibration equations showed that the described method could expand the measurement limits of digital dynamometer DOU-3-1I by more than $50 \%$ (or more precisely, by $53.44 \%$ ), i.e. the maximum destruction load that can be recorded with the digital dynamometer of the test bench is no longer 1 but $1.5 \mathrm{kN}$. Such expansion of destruction load measurement limits was quite enough to test the bending strength of all sod peat samples.

\section{Conclusion}

To conclude and prove the practical application of the method described above, we provide the results of testing sod peat bending strength in a laboratory environment, when sod peat was moulded with a screw press having replaceable screws with 50 and $110 \mathrm{~mm}$ pitches and a set of replaceable tips providing pressure coefficients of 2.96, 2.22, 0.95 [9] (Fig. 4, $5)$. 


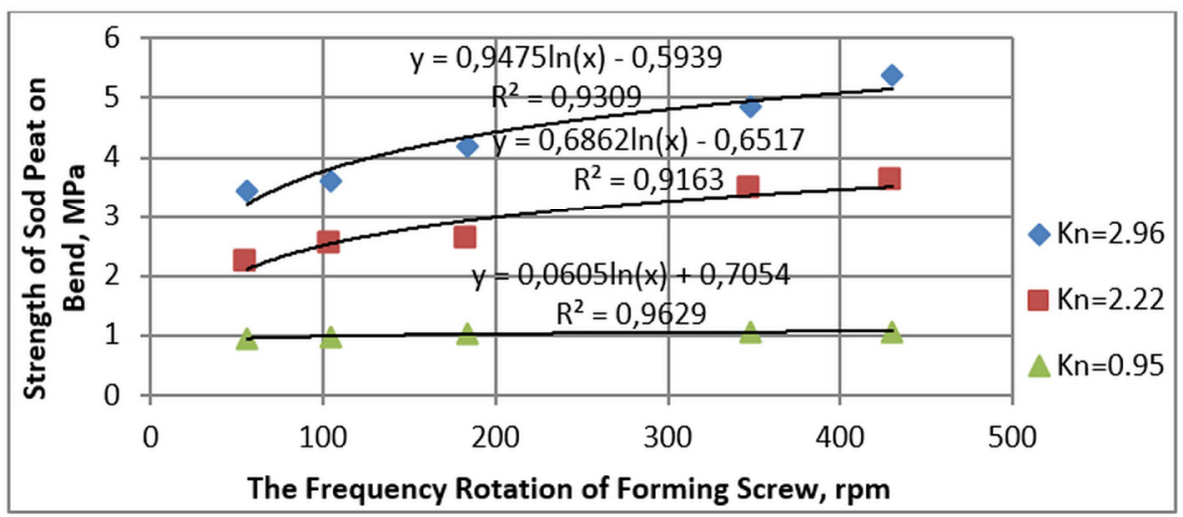

Fig. 4. The dependence of sod peat strength on the screw rotation speed in press moulding with 110 $\mathrm{mm}$ pitch for pressure coefficients $K n$.

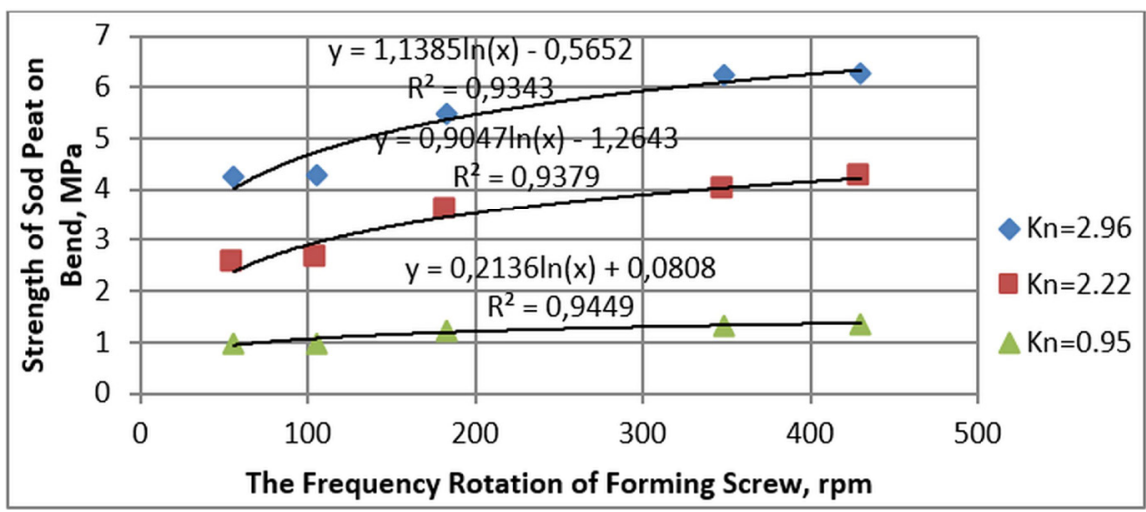

Fig. 5. The dependence of sod peat strength on the screw rotation speed in press moulding with 50 $\mathrm{mm}$ pitch for pressure coefficients $K n$.

It should be noted that the available equipment would do it impossible to perform experiments to determine the strength characteristics of moulded peat products if the way to expand the measurement limits of a digital dynamometer could not be found.

\section{Inferences}

The design and rational use of milling machines will reduce the peat loss during production [4] and reduce the specific energy consumption without losing a sod density and, consequently, its strength [10]. At the same time, to achieve rational operating modes of milling-forming machines is impossible without operational sod quality surveillance [9]. The test bench proposed to test destruction of the sod bending strength can help obtain very reliable information with small dimensions and simplicity of its design. Besides, the expansion of the limits of destruction load measurements by more than $50 \%$ makes it possible to check all possible moulded peat products with the range of available equipment, which the authors have done successfully. The results of sod peat tests for strength show that milling machines work efficiently, if we provide a screw rotation speed of 220-320 rpm, use screws with a pitch close to the half of its diameter, and the ratio of the number and diameter of moulding tips to create a pressure coefficient being close to 2.22 [9]. 


\section{References}

1. O. Misnikov, E3S Web Conf., 21, 01020 (2017)

2. V.V. Panov, O.S. Misnikov, Min. J., 7, 108-112 (2015)

3. O. Misnikov, E3S Web Conf., 41, 01046 (2018)

4. E. A. Kremcheev, D.A. Kremcheeva, Indian Journal of Science and Technology, 9:12, 89525 (2016)

5. K. Singh, AEP Public Land Management, 9, 1-14 (2016)

6. J. Korpi, Proceed.13-th Int. Peat Cong., Tullamore, Ireland, 1, 120-122 (2008)

7. R. K. Singh, A. Das, Fuel Processing Technology, 115, 71-78 (2013)

8. Z. N. Gholipour, M. A. Zarringhalam, S. Dorosti, Petroleum \& Coal, 56:2, 188-195 (2014)

9. A. Yablonev, A. Guseva, E3S Web Conf., 105, 01033 (2019)

10. R. Lindsay, R. Birnie, IUCNUK Committee Peatland Programme, 6, 1-5 (2014) 